\title{
Thiocoumarin Caged Nucleotides: Synthetic Access and Their Photophysical Properties
}

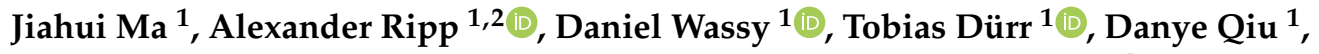 \\ Markus Häner ${ }^{1}$, Thomas Haas ${ }^{1}$, Christoph Popp ${ }^{1}$, Dominik Bezold ${ }^{1} \mathbb{D}$, Sabine Richert ${ }^{3} \mathbb{D}$, \\ Birgit Esser 1,2 and Henning J. Jessen 1,2,*iD \\ 1 Institute of Organic Chemistry, University of Freiburg, Albertstr. 21, 79104 Freiburg, Germany; \\ jiahui.ma@oc.uni-freiburg.de (J.M.); alexander.ripp@livmats.uni-freiburg.de (A.R.); \\ daniel.wassy@oc.uni-freiburg.de (D.W.); tobias.duerr@oc.uni-freiburg.de (T.D.); \\ danyeqiu@gmail.com (D.Q.); markus.haener@oc.uni-freiburg.de (M.H.); \\ thomas.haas@oc.uni-freiburg.de (T.H.); christoph.popp@ocbc.uni-freiburg.de (C.P.); \\ dominik.bezold@ocbc.uni-freiburg.de (D.B.); besser@oc.uni-freiburg.de (B.E.) \\ 2 Cluster of Excellence livMatS @ FIT-Freiburg Center for Interactive Materials and Bioinspired Technologies, \\ University of Freiburg, Georges-Köhler-Allee 105, 79110 Freiburg, Germany \\ 3 Institute of Physical Chemistry, University of Freiburg, Albertstr. 21, 79104 Freiburg, Germany; \\ sabine.richert@physchem.uni-freiburg.de \\ * Correspondence: henning.jessen@oc.uni-freiburg.de; Tel.: +49-(761)-203-6073
}

Academic Editor: Marie Migaud

Received: 29 October 2020; Accepted: 13 November 2020; Published: 15 November 2020

check for updates

\begin{abstract}
Photocages have been successfully applied in cellular signaling studies for the controlled release of metabolites with high spatio-temporal resolution. Commonly, coumarin photocages are activated by UV light and the quantum yields of uncaging are relatively low, which can limit their applications in vivo. Here, syntheses, the determination of the photophysical properties, and quantum chemical calculations of 7-diethylamino-4-hydroxymethyl-thiocoumarin (thio-DEACM) and caged adenine nucleotides are reported and compared to the widely used 7-diethylamino-4-hydroxymethyl-coumarin (DEACM) caging group. In this comparison, thio-DEACM stands out as a phosphate cage with improved photophysical properties, such as red-shifted absorption and significantly faster photolysis kinetics.
\end{abstract}

Keywords: thio-DEACM; photocage; nucleotides; photochemistry

\section{Introduction}

Chemists had started to use light as an external trigger around the 1970s to control the release of various molecules [1,2]. Light triggers relying on photocleavable protecting groups (PPGs or photocages) have shown significant utility for the study and control of biological processes in living cells and tissues [1]. Among the numerous available photocages, one of the most widely applied classes is based on the coumarin scaffold. Coumarin derivatives exhibit a range of appealing benefits as caging groups, for example, easy synthesis, high biocompatibility [3], flexibility of structural modifications [4] with tunable spectral properties [5], while the mechanism of photocleavage is well understood [6-8]. Attractive applications of coumarin derivatives in chemical biology include cell signaling molecules [4,9-11], neurotransmitters [12-15], peptides [16], and photosensitizers [17]. Furthermore, additional photocages based on the coumarin scaffold are being developed, such as derivatives with modifications at the 2-position [18-21], azetidinyl substituents at the 7-position [22], $\pi$-extension at the 3-position [5], and replacement of the oxygen with sulfur on the lactone carbonyl group [23]. 
In particular, photocaging of nucleotides as cellular signals is an important area of research. Adenosine 5'-triphosphate (ATP) is not only the essential intracellular energy source, but is also involved in many physiological processes [24]. Not surprisingly, different photocages were installed on ATP, for example, 2-nitrobenzyl (commercially available) [2], 1-(2-nitro) phenylethyl [2], P3-3',5'-dimethoxybenzoin ester (DMB) [25,26], p-hydroxyphenacyl (pHP) [27], [7-(dimethylamino)coumarin-4-yl]methyl (DMACM) [28], and [7-(diethylamino)coumarin-4-yl]methyl (DEACM) [29]. Most of these caged ATP molecules are activated by UV light, which impaired their applications in cells and tissue, apart from limited cellular uptake.

Thionated coumarins were first reported in 2012 [30] as photocages for carboxylates (Figure 1). Compared to the corresponding carbonyl precursors, they show a considerable bathochromic shift (between 47 and $73 \mathrm{~nm}$ ) in absorption and faster uncaging kinetics upon irradiation. In 2013, Jullien's group also used the strategy to replace oxygen by sulfur, this time on the well-known DEACM photocage [31]. The red-shift in absorption was further increased because of the strong electron donating $\mathrm{Et}_{2} \mathrm{~N}$ group in the 7-position. Additionally, in their study a carboxylate was released (benzoate), and blue light was used to trigger the reaction. 7-diethylamino-4-hydroxymethyl-thiocoumarin (thio-DEACM) was also used to cage a cyclofen-OH analogue (Figure 1, tcInd) as a carbamate and applied in living zebrafish embryos [23]. This was the first report where thio-DEACM was applied in an in vivo study. In 2015, benzoic acid-protected thio-DEACM was also used in upconverting nanoparticles (UCNPs)-assisted photochemistry [32]. In 2019, thio-DEACM was introduced to trimethoprim (TMP) [33], again for the caging of carbamates. After uncaging with low-intensity light, TMP was able to activate Cas9. Temporal and spatial control over Cas9 activity by photocaged TMP was demonstrated, and importantly the photolysis was nontoxic to both mammalian and bacterial cells.

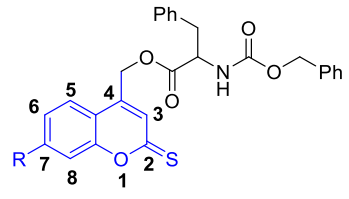

$\mathrm{R}=\mathrm{CH}_{3}, \mathrm{OCH}_{3}$ 2012

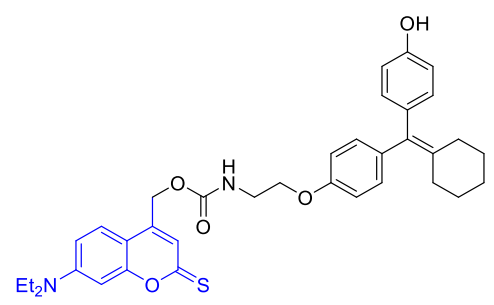

tclnd

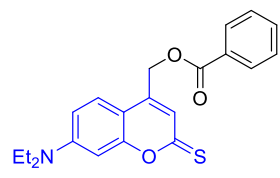

NdiEt-tcBA 2013

Figure 1. Structures of previously reported 7-diethylamino-4-hydroxymethyl-thiocoumarin (thio-DEACM) (blue) caged molecules with the year of publication indicated underneath the structure.

These reports underline the attractive features of thio-DEACM as caging group, such as rapid blue-cyan light responsiveness and avoiding UV irradiation of cells in combination with the absence of toxicity of the released photocage. However, unlike the widely used nitrobenzyl (NB) photocage or several coumarin derivatives, particularly DEACM, thio-DEACM has received only little attention. This may be due to impediments regarding its synthesis and also its incorporation into target structures, particularly phosphoric anhydrides, which have not been caged with thio-DEACM yet. To evaluate the utility of thio-DEACM for caging of phosphoric anhydrides, we synthesized several thio-DEACM-caged adenine nucleotides and compared them to their DEACM-caged siblings. We found that orthogonal 
uncaging of the different photocages with visible light is possible (Scheme 1), which will enable applications of thio-DEACM-caged phosphates in cell and tissue studies in the future.
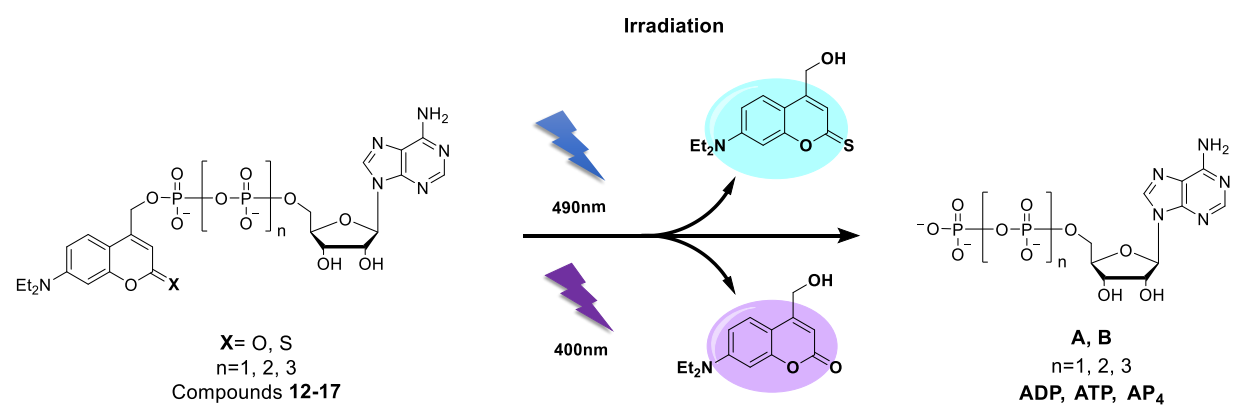

Scheme 1. Schematic representation of the uncaging process and the orthogonal features of 7-diethylamino-4-hydroxymethyl-coumarin (DEACM) and thio-DEACM. Irradiation with $490 \mathrm{~nm}$ light will liberate thio-DEACM from nucleotide A. Irradiation with $400 \mathrm{~nm}$ light will liberate DEACM from nucleotide B. Irradiation with $490 \mathrm{~nm}$ light and $400 \mathrm{~nm}$ light will liberate both A and B.

\section{Results}

\subsection{Chemistry}

DEACM alcohol 4 was synthesized over three steps in $56 \%$ overall yield from commercially available starting materials following a previously reported procedure (Supplementary Scheme S1) [34]. Afterwards, thio-DEACM was obtained by replacing the carbonyl oxygen with sulfur using Lawesson's reagent [30] in 65\% yield (Scheme 2A). Subsequently, thio-DEACM was incorporated into phosphorodiamidite 8 [35] (Scheme 2B) giving phosphoramidite (P-amidite) 9, which serves as a general precursor for phosphoric anhydride synthesis following previously published $\mathrm{P}(\mathrm{III})-\mathrm{P}(\mathrm{V})$ anhydride synthesis protocols [36]. Overall, P-amidite 9 was obtained in eight steps and 12\% yield.

In brief, P-amidite 9 was utilized in the synthesis of modified phosphoric anhydrides generated on unprotected nucleotides (here: AMP, ADP, ATP) via a coupling, oxidation and deprotection strategy [36]. 5-(Ethylthio)-1H-tetrazole (ETT) was used as an activator for the coupling step, giving $\mathrm{P}(\mathrm{III})-\mathrm{P}(\mathrm{V})$ intermediates 10 (Scheme 2C). In the same flask, meta-chloroperbenzoic acid ( $m$ CPBA) was used for oxidation to a $\mathrm{P}(\mathrm{V})-\mathrm{P}(\mathrm{V})$ anhydride 11. After complete oxidation, piperidine was added to remove the Fm group. This represents a convenient synthesis, as all three steps can be conducted in one flask and proceed rapidly. The crude products 12-14 were precipitated as sodium salts by addition of a $0.5 \mathrm{M}$ $\mathrm{NaClO}_{4}$ solution in acetone $[37,38]$ and purified by strong anion-exchange (SAX) chromatography with an $\mathrm{NH}_{4} \mathrm{HCO}_{3}$ - buffer gradient. The product containing fractions were combined, lyophilized, and then again purified by reverse phase chromatography (RP-C18AQ) with acetonitrile in $\mathrm{H}_{2} \mathrm{O}$ with triethylammonium acetate (TEAA) buffer as eluent. We observed formation of DEACM-caged ADP (15), ATP (16) and $\mathrm{AP}_{4}$ (17) as minor impurities. Apparently, during the oxidation step with $m C P B A$, thio-DEACM was partially oxidized to DEACM. After purification, DEACM- and thio-DEACM-caged nucleotides 12-17 were obtained. Even though the product nucleotides only differ in one atom (O vs. S on DEACM or thio-DEACM), they were readily separated by preparative SAX chromatography. In this regard, the minor side-reaction was useful to also obtain DEACM-caged nucleotides 15-17 for direct comparison with thio-DEACM-modified analogues 12-14 as discussed in the next section. 
A.

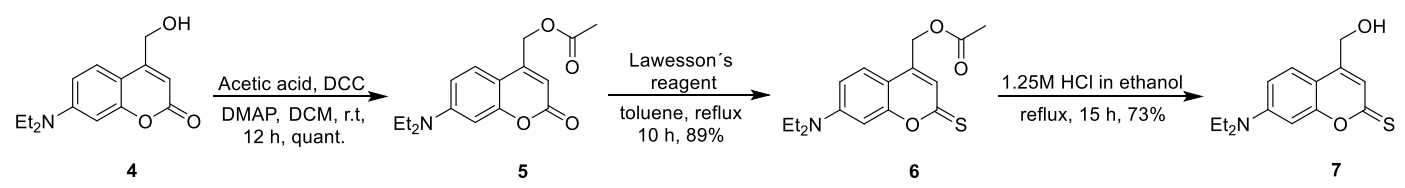

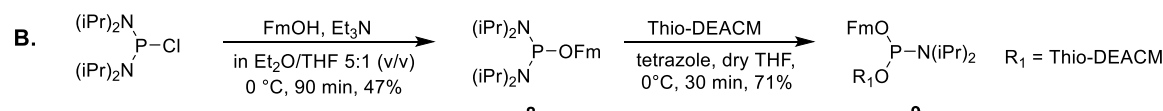

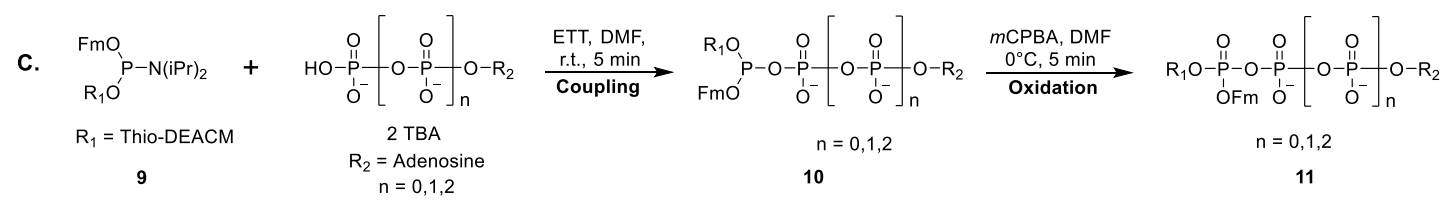

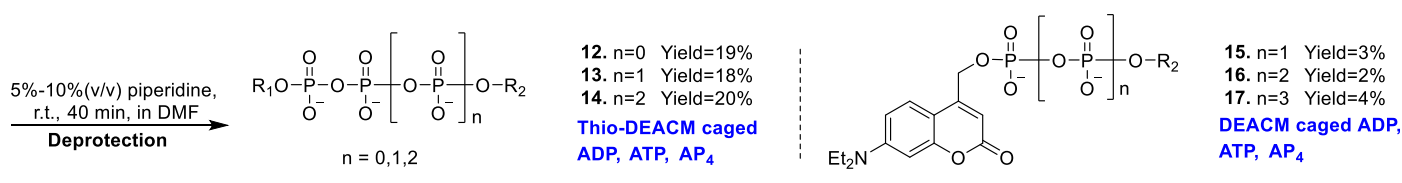<smiles>[Y]c1cc(=S)oc2cc(N=[R16])ccc12</smiles>

Scheme 2. (A) Synthesis of thio-DEACM 7. (B) Synthesis of P-amidite 9. (C) Synthesis of thio-DEACM-caged nucleotides. One-flask synthesis of thio-DEACM-caged nucleotides. The caged nucleotides are obtained as triethylammonium (TEA) salts after purification. The number of counterions was determined by ${ }^{1} \mathrm{H}$ NMR (not indicated in this scheme).

\subsection{Photophysical Properties}

To compare the photophysical properties of DEACM 4, thio-DEACM 7 and their corresponding caged phosphate esters 12-14 and 15-17, absorption maxima $\left(\lambda_{a b s}^{\text {max }}\right)$, fluorescence maxima $\left(\lambda_{f l}^{\text {max }}\right)$, molar absorption coefficients $\varepsilon\left(\lambda_{a b s}^{\max }\right)$, and fluorescence quantum yields $\left(\Phi_{\mathrm{fl}}\right)$ were measured. Moreover, density functional theory (DFT) calculations were performed to predict absorption maxima of the alcohols, which are in good agreement with the experimental values. The values are listed in Table 1 (Experimental details, Supplementary Figures S1-S7).

Table 1. $\lambda_{a b s}^{\max }$ : absorption maximum, $\lambda_{f l}^{\max }$ : fluorescence maximum, $\varepsilon\left(\lambda_{a b s}^{\max }\right)$ : molar absorption coefficient, $\Phi_{\mathrm{fl}}$ : fluorescence quantum yield.

\begin{tabular}{|c|c|c|c|c|}
\hline Compound & $\lambda_{a b s}^{\max }[\mathrm{nm}]$ & $\lambda_{f l}^{\max }[\mathrm{nm}]$ & $\varepsilon\left(\lambda_{a b s}^{\max }\right)\left[\mathrm{mM}^{-1} \mathrm{~cm}^{-1}\right]$ & $\Phi_{\mathrm{fl}}(\%)$ \\
\hline DEACM (4) & $375^{1}\left(374^{6}\right)$ & $472^{1}$ & $16.8^{1}$ & $25.6^{1}$ \\
\hline Thio-DEACM (7) & $457^{1}\left(435^{6}\right)$ & $534^{1}$ & $19.2^{1}$ & $<1^{1}$ \\
\hline DEACM ADP $(\mathbf{1 5})^{2}$ & $392^{3}$ & 498 & $16.3^{4}$ & $17.7^{5}$ \\
\hline Thio-DEACM ADP (12) ${ }^{2}$ & $480^{3}$ & 572 & $13.5^{4}$ & $2.7^{5}$ \\
\hline $\operatorname{DEACM} \operatorname{ATP}(\mathbf{1 6})^{2}$ & $390^{3}$ & 498 & $25.9^{4}(24.7)^{7}$ & $12.4^{5}$ \\
\hline Thio-DEACM ATP (13) ${ }^{2}$ & $480^{3}$ & 568 & $25.6^{4}(4.6)^{7}$ & $2.2^{5}$ \\
\hline $\mathrm{DEACM} \mathrm{AP}_{4}(\mathbf{1 7})^{2}$ & $392^{3}$ & 498 & $16.6^{4}$ & $13.3^{5}$ \\
\hline Thio-DEACM AP $4(\mathbf{1 4})^{2}$ & $480^{3}$ & 570 & $15.9^{4}$ & $2.2^{5}$ \\
\hline
\end{tabular}

\footnotetext{
${ }^{1} \mathrm{In} \mathrm{MeOH}$ solution. Concentration: $50 \mu \mathrm{M}(\mathrm{abs}), 12.5 \mathrm{nM}(\mathrm{fl}), 20 \mu \mathrm{M}(\varepsilon), 100 \mu \mathrm{M}\left(\boldsymbol{\Phi}_{\mathrm{fl}}\right) .{ }^{2}$ In ultrapure water.

${ }^{3}$ Concentration: $50 \mu \mathrm{M} .{ }^{4}$ Concentration: $20 \mu \mathrm{M} .{ }^{5}$ Concentration: $100 \mu \mathrm{M}$. ${ }^{6}$ Values from density functional theory

(DFT) calculation with water as solvent. ${ }^{7} \varepsilon(400 \mathrm{~nm})$.
} 
Thio-DEACM 7 and thio-DEACM-caged nucleotides 12-14 showed a bathochromic shift in absorption and emission compared to DEACM, as expected [31]. Additionally, the fluorescence quantum yields are significantly reduced. As the mechanism of photocleavage requires heterolytic bond scission in the excited state, radiative deactivation by fluorescence impairs this process, as do other non-radiative deactivation processes [7]. The lower deactivation by fluorescence, as found for the thio-DEACM cage, boded well for increased quantum yields of uncaging, even though the dark deactivation pathways are not known.

To provide a first theoretical explanation for the experimental results regarding the bathochromic shifts and to explore additional interesting modifications on the coumarin backbone, density functional theory (DFT) calculations were conducted (Figure 2). We also included a selenium substituted coumarin in the calculations and predict a further red-shift in comparison to DEACM and thio-DEACM.

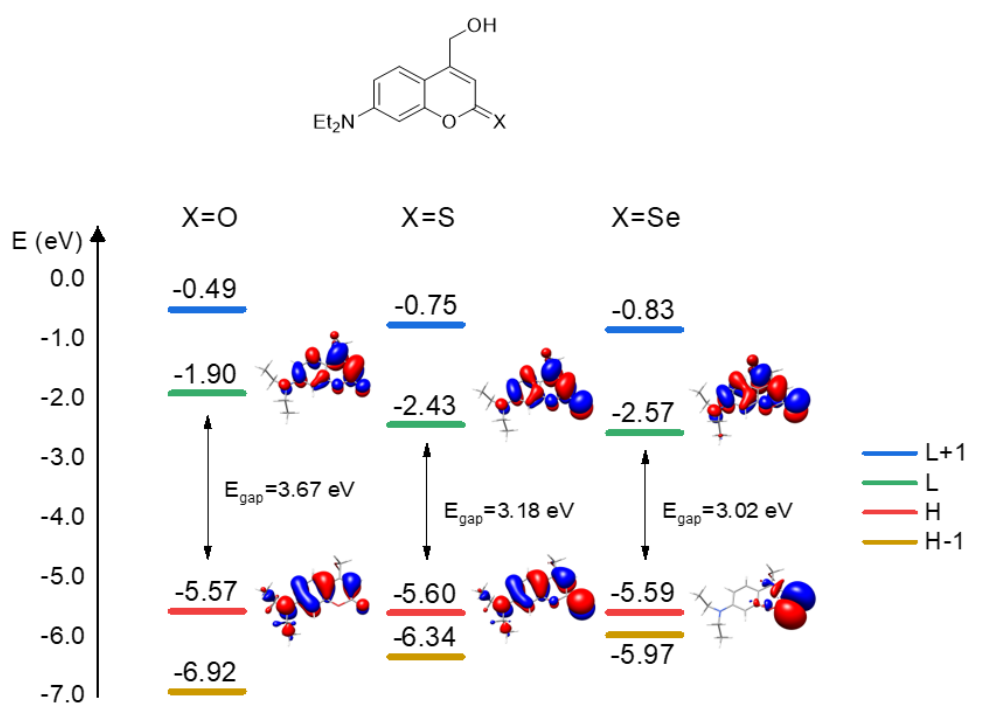

Figure 2. Investigated structures and frontier molecular orbital energies from single-point calculations on the B3LYP/def2-TZVPP//B3LYP/def2-TZVPP level of theory $(\mathrm{H}=$ highest occupied molecular orbitals (HOMO), L = lowest occupied molecular orbitals (LUMO)).

Kim and Lee previously investigated the heavy atom effect of selenium in phenoselazines [39]. They screened several density functionals with varying Fock exchange and found B3LYP to best reproduce the experimental spectra. Thus, the B3LYP $[40,41]$ hybrid functional with the def2-TZVPP [42,43] basis set was used in our studies. A relatively large basis set with extra polarization functions is needed to describe the rather diffuse electrons of selenium and sulfur. The Polarizable Continuum Model (PCM) for water, as implemented in Gaussian16 was used in all calculations.

The values represented in Figure 2 show a clear trend in the orbital energies from oxygen to selenium. The energy of the lowest unoccupied molecular orbitals (LUMOs) decreases from -1.90 (oxygen) to $-2.57 \mathrm{eV}$ (selenium), whereas the energies of the highest occupied molecular orbitals (HOMOs) remain the same within the chalcogens (around $-5.60 \mathrm{eV}$ ). This leads to a decrease in the energy gap from 3.67 (oxygen) to $3.02 \mathrm{eV}$ (selenium).

Time dependent (TD)-DFT calculations provided absorption spectra of the photocages (Supplementary Figure S8). The first 15 singlet excitations were calculated on the TD-B3LYP/def2-TZVPP level of theory. The resulting longest wavelength absorption maxima are $374 \mathrm{~nm}(\mathrm{O}), 435 \mathrm{~nm}(\mathrm{~S})$ and $457 \mathrm{~nm}$ (Se). These are all attributed to HOMO to LUMO excitations. The same trend observed in the frontier molecular orbital energies was also found here, namely a decreasing band gap from $\mathrm{O}$ to Se and a red-shift in absorption. 


\subsection{Photolysis Studies}

The stability of caged molecules in water in the dark is very important in photolysis studies and furthermore in biological experiments. This is particularly the case for potentially labile phosphoric anhydrides as present in the caged nucleotides 12-14. Thus, the stability of thio-DEACM-caged nucleotides in the dark at different concentrations $(0.1 \mathrm{mM}, 0.5 \mathrm{mM}, 1 \mathrm{mM})$ was evaluated over a period of $24 \mathrm{~h}$ by HPLC-MS. All of the analyzed molecules were found to be stable (Supplementary Figure S9).

Next, uncaging experiments were performed using a commercially available LED setup with two distinct wavelengths (Mightex ${ }^{\circledR}$ High-Power LED Collimator Sources, Martinsried, Germany; for the $400 \mathrm{~nm}$ LED: $48 \mathrm{~mm}$ aperture, typical output power $265 \mathrm{~mW}$; for the $490 \mathrm{~nm}$ LED: $22 \mathrm{~mm}$ aperture, typical output power $140 \mathrm{~mW}$ ). The emission spectra of the LEDs were measured with an (Ocean Insight ${ }^{\circledR}$ USB 2000+, Ostfildern, Germany) miniature spectrometer (Figure 3a), showing narrow emission bands with no significant overlap. The actual center wavelength for the $400 \mathrm{~nm}$ LED is $396 \mathrm{~nm}$, and full width at half maximum (FWHM) is $13 \mathrm{~nm}$. The actual center wavelength for the $490 \mathrm{~nm}$ LED is $494 \mathrm{~nm}$, and FWHM is $29 \mathrm{~nm}$. The LED emissions match well with the absorption spectra of the two different photocaged molecules (Figure 3).

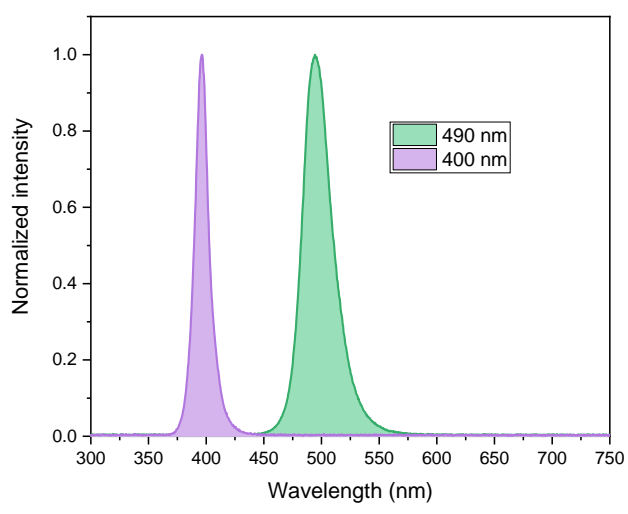

(a)

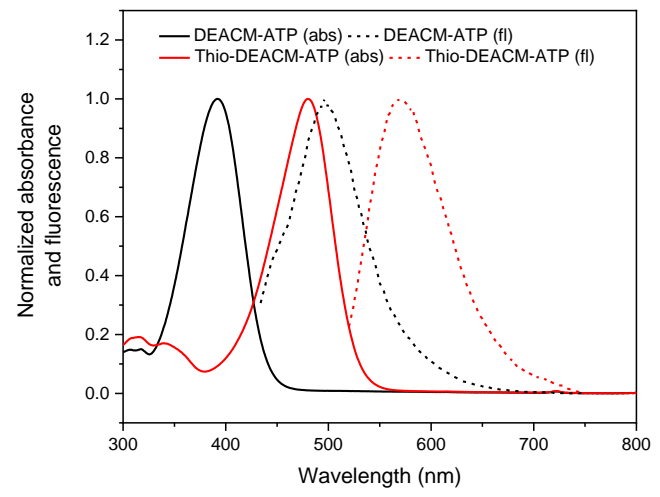

(b)

Figure 3. (a) The spectra of the LED light sources (400 nm and $490 \mathrm{~nm})$, (b) normalized absorbance (abs) and emission (fl) spectra of DEACM- and thio-DEACM-caged ATP in $\mathrm{H}_{2} \mathrm{O}$.

Subsequently, $1 \mathrm{mM}$ sample solutions of the caged nucleotides were prepared in ultrapure water in glass vials and placed $3 \mathrm{~cm}$ away from the light source (Experimental setup, Supplementary Figure S10). Irradiation with different light intensities and for different time intervals were done as reported below. Afterwards, the samples were directly analyzed by HPLC-UV (Thermo, Darmstadt, Germany). The percentage of remaining caged compound was calculated as well as the uncaging ratio. Released nucleotides were plotted according to the uncaging ratios. (Supplementary Figures S13-S15, S17; Tables S4-S7).

The uncaging kinetics of thio-DEACM-modified ATP 13 were measured at different wavelengths, light intensities, and time points and compared to DEACM-caged ATP 16. At $490 \mathrm{~nm}$, three different light intensities were used for the photolysis experiments. Thio-DEACM-caged ATP is converted to ATP at high rates, even with $10 \%$ of the nominal light intensity. The process was also monitored by ${ }^{31} \mathrm{P} \mathrm{NMR}$ spectroscopy (Figure 4 and Supplementary Figure S11). From NMR monitoring, thio-DEACM-caged ATP 13 was cleanly uncaged upon irradiation, and ATP was the only phosphorous-containing product. Since the sensitivity of NMR spectroscopy is limited, capillary electrophoresis (CE) —MS analysis (Supplementary Figure S12) was also applied to identify the released ATP. 


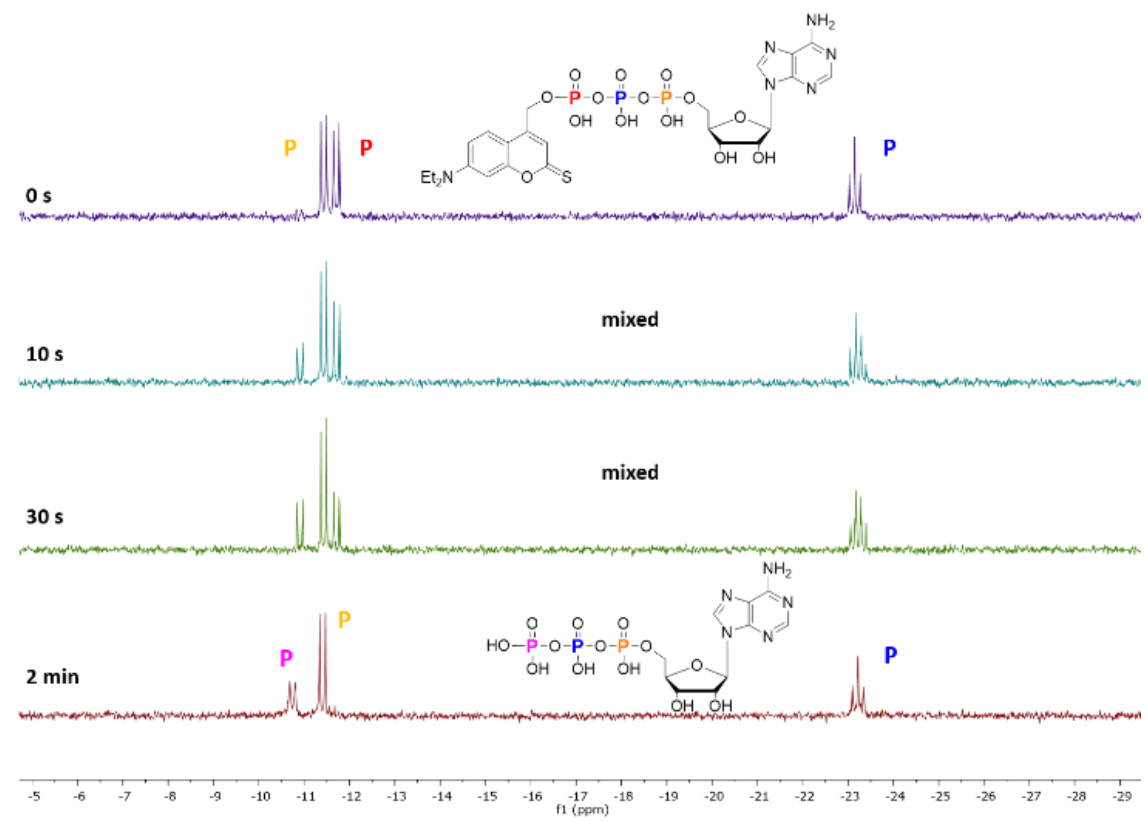

Figure 4. Uncaging process of thio-DEACM-caged ATP monitored by ${ }^{31} \mathrm{P}$ NMR spectroscopy $(2 \mathrm{mg} / \mathrm{mL}$ in $\mathrm{D}_{2} \mathrm{O}$, irradiation at $490 \mathrm{~nm}, 50 \%$ light intensity).

To study differences in the performance of DEACM- and thio-DEACM-caged molecules in response to irradiation at different wavelengths and intensities, the photolysis of DEACM-caged ATP 16 was evaluated in parallel. At $490 \mathrm{~nm}$, no removal of the DEACM cage was observed (Supplementary Figure S16), since DEACM-caged ATP 16 does not absorb light at this wavelength (Figure 3b, black line). Thus, only thio-DEACM-caged ATP 13 can be uncaged with $490 \mathrm{~nm}$ light (Figure 5a).

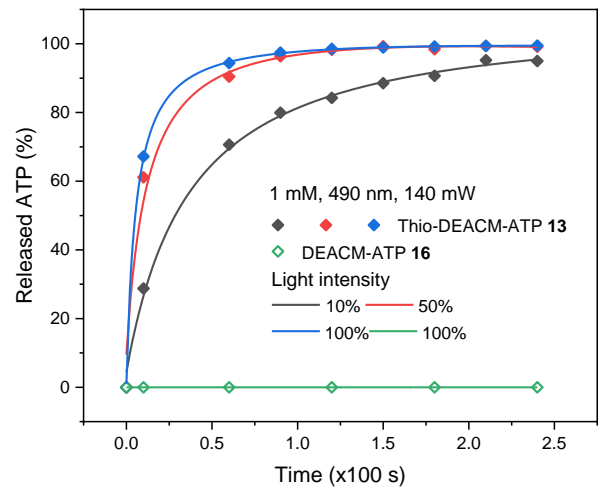

(a)

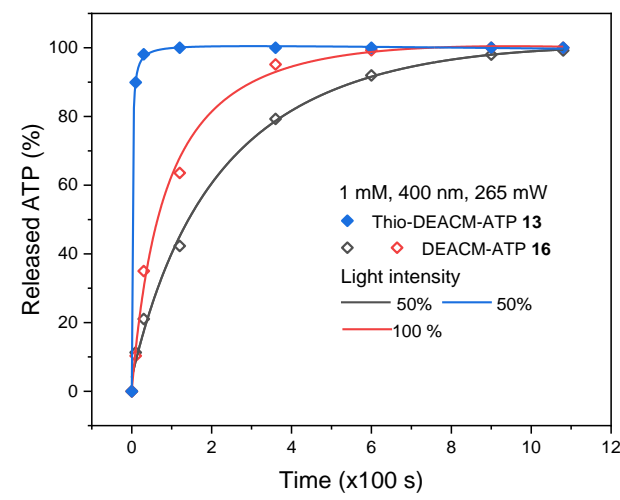

(b)

Figure 5. (a) Photolysis of thio-DEACM-caged ATP $13(1 \mathrm{mM})$ at $490 \mathrm{~nm}$ with $10 \%, 50 \%, 100 \%$ of the nominal LED light intensity over $4 \mathrm{~min}$ and photolysis of DEACM-caged ATP $16(1 \mathrm{mM})$ at $490 \mathrm{~nm}$ with 100\% light intensity over $4 \mathrm{~min}$ (140 mW LED). (b) Photolysis of thio-DEACM-caged ATP 13 $(1 \mathrm{mM})$ at $400 \mathrm{~nm}$ with $50 \%$ light intensity and photolysis of DEACM-caged ATP $16(1 \mathrm{mM})$ at $400 \mathrm{~nm}$ with $50 \%$ and $100 \%$ light intensity over $18 \mathrm{~min}$ (265 mW LED).

To photolyze DEACM-caged ATP 16, a 400 nm LED was used, which emits close to its absorption maximum (Figure 3). With $400 \mathrm{~nm}$ light irradiation, the photolysis of $\mathbf{1 6}$ was complete in ca. $18 \mathrm{~min}$ using $50 \%$ or $100 \%$ of the nominal LED light intensity at $1 \mathrm{mM}$ concentration (Figure $5 \mathrm{~b}$, black and red lines). The same conditions applied to thio-DEACM-caged ATP 13 (50\% light intensity, $400 \mathrm{~nm}$ ) led to complete ATP release within $30 \mathrm{~s}$ (Figure $5 \mathrm{~b}$, blue line), despite the fact that thio-DEACM-caged ATP has lower absorbance at this wavelength (Table 1). 
The same behavior was observed also for caged ADPs 12 and 15 (Figure 6a) and caged $\mathrm{AP}_{4} \mathrm{~s}$ [44] 14 and 17 (Figure $6 \mathrm{~b}$ ). This underscores the remarkable utility of thio-DEACM as a phosphate caging group.

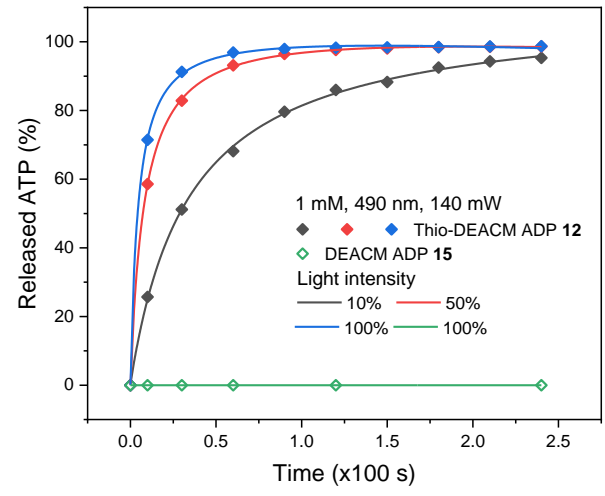

(a)

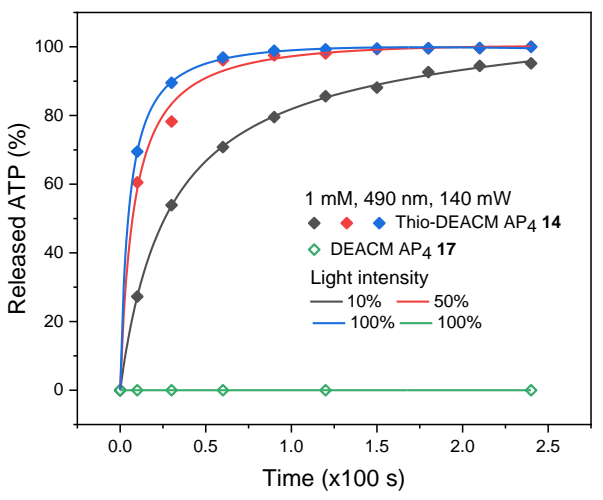

(b)

Figure 6. (a) and (b) Photolysis of thio-DEACM-caged ADP and $\mathrm{AP}_{4} \mathbf{1 2}, \mathbf{1 4}(1 \mathrm{mM})$ at $490 \mathrm{~nm}$ with $10 \%$, $50 \%, 100 \%$ light intensity over $4 \mathrm{~min}$ and photolysis of DEACM-caged ADP and $\mathrm{AP}_{4} \mathbf{1 5}, \mathbf{1 7}(1 \mathrm{mM})$ at $490 \mathrm{~nm}$ with $100 \%$ light intensity over $4 \mathrm{~min}$ (140 mW LED).

Overall, the vastly different reaction rates and light responsiveness indicated that DEACMand thio-DEACM-caged molecules can be uncaged orthogonally. A mixture of DEACM- and thio-DEACM-caged ATP 13 and 16 proved this selectivity, as only 13 was cleaved upon irradiation at $490 \mathrm{~nm}$ (Figure 7a). After full release from thio-DEACM-caged ATP 13, irradiation with the $400 \mathrm{~nm}$ LED released additional ATP from DEACM-caged ATP 16. This process was monitored by HPLC-UV (Figure 7a). Again, it is noteworthy that despite the fact that the compounds only differ in a single atom (S vs. O), baseline separation was readily achieved using reversed phase HPLC. Likewise, the orthogonal uncaging could also be conducted with thio-DEACM- and DEACM-caged ADP 12 and 15 (Figure $7 \mathrm{~b}$ ) and $\mathrm{AP}_{4} 14$ and 17 (Figure 7c).

Furthermore, when DEACM-caged ATP 16 and thio-DEACM-caged ADP 12 were mixed, $490 \mathrm{~nm}$ light can selectively liberate ADP, followed by release of ATP at $400 \mathrm{~nm}$ (Figure 8a). The DEACM and thio-DEACM photocages enable a very clean orthogonal uncaging at the selected wavelengths. Successive and clean uncaging of DEACM-caged $\mathrm{AP}_{4} 17$ and thio-DEACM-caged ADP 12, DEACM-caged $\mathrm{AP}_{4} 17$ and thio-DEACM-caged ATP 13 were also possible (Figure 8b,c).

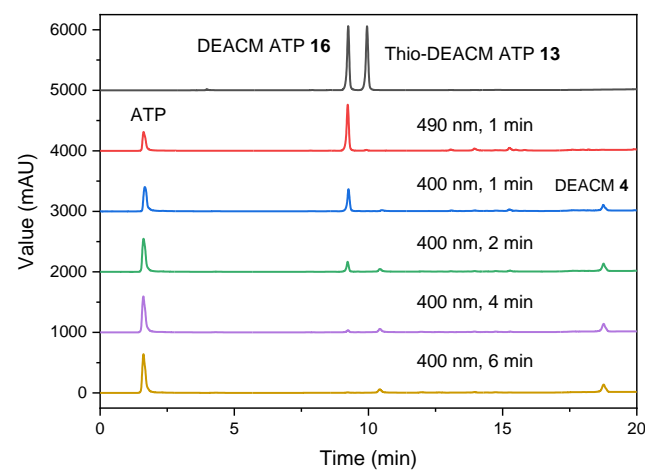

(a)

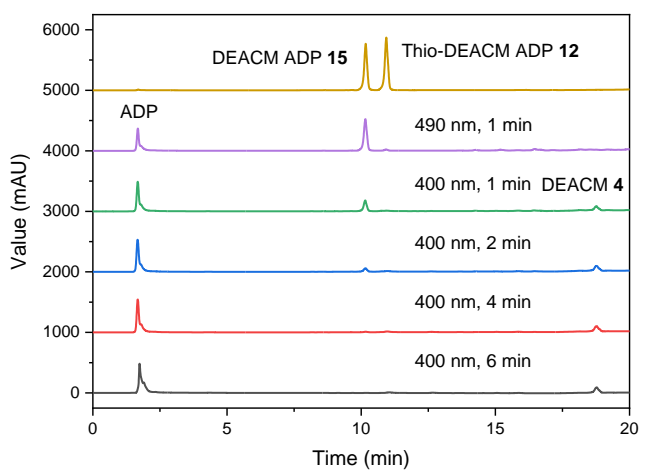

(b)

Figure 7. Cont. 


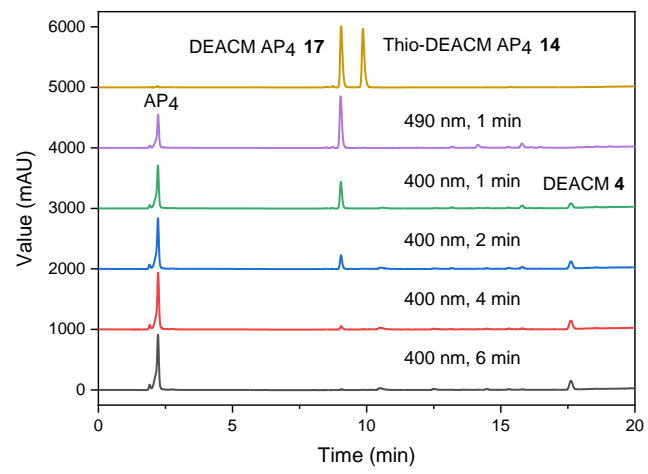

(c)

Figure 7. (a) Photolysis of a mixture of thio-DEACM- and DEACM-caged ATPs 13 and 16 (0.5 mM) irradiated at $490 \mathrm{~nm}$ with $100 \%$ light intensity over $1 \mathrm{~min}$ and then at $400 \mathrm{~nm}$ with $100 \%$ light intensity for $6 \mathrm{~min}$ (265 mW LED) monitored by HPLC-UV. (b) Photolysis of a mixture of thio-DEACM- and DEACM-caged ADPs 12 and $15(0.5 \mathrm{mM})$ irradiated at $490 \mathrm{~nm}$ with $100 \%$ light intensity over $1 \mathrm{~min}$ and then at $400 \mathrm{~nm}$ with 100\% light intensity over $6 \mathrm{~min}$ (265 mW LED) monitored by HPLC-UV. (c) Photolysis of a mixture of thio-DEACM- and DEACM-caged $\mathrm{AP}_{4} \mathrm{~s} 14$ and $17(0.5 \mathrm{mM})$ irradiated at $490 \mathrm{~nm}$ with $100 \%$ light intensity over $1 \mathrm{~min}$ and then at $400 \mathrm{~nm}$ with $100 \%$ light intensity over $6 \mathrm{~min}$ (265 mW LED) monitored by HPLC-UV. UV detector with $254 \mathrm{~nm}$ detection.

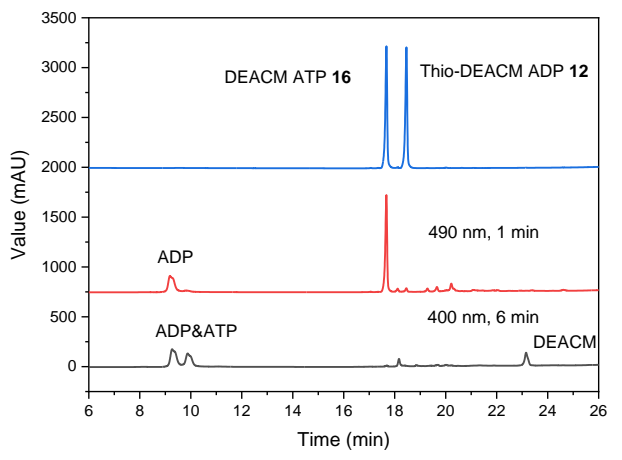

(a)

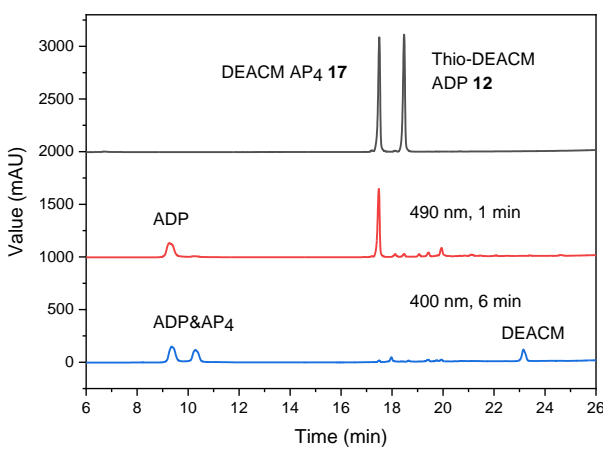

(b)

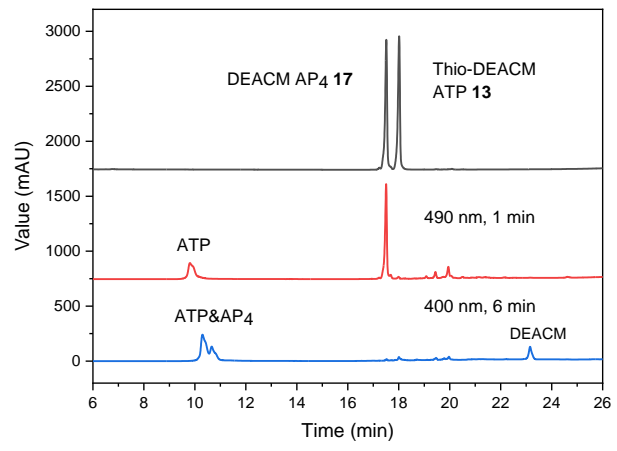

(c)

Figure 8. (a) Photolysis of DEACM-caged ATP 16 and thio-DEACM-caged ADP 12 as a mixture $(0.5 \mathrm{mM})$ at $490 \mathrm{~nm}$ with $100 \%$ light intensity over $1 \mathrm{~min}$ and then at $400 \mathrm{~nm}$ with $100 \%$ light intensity over 6 min (265 mW LED) monitored by HPLC-UV. (b) Photolysis of DEACM-caged $\mathrm{AP}_{4} 17$ and thio-DEACM-caged ADP 12 as a mixture $(0.5 \mathrm{mM})$ at $490 \mathrm{~nm}$ with $100 \%$ light intensity over $1 \mathrm{~min}$ and then at $400 \mathrm{~nm}$ with 100\% light intensity over $6 \mathrm{~min}$ (265 mW LED) monitored by HPLC-UV. (c) Photolysis of DEACM-caged $\mathrm{AP}_{4} \mathbf{1 7}$ and thio-DEACM-caged ATP 13 as a mixture $(0.5 \mathrm{mM})$ at $490 \mathrm{~nm}$ with $100 \%$ light intensity over $1 \mathrm{~min}$ and then at $400 \mathrm{~nm}$ with $100 \%$ light intensity over $6 \mathrm{~min}$ (265 mW LED) monitored by HPLC-UV. UV detector with $254 \mathrm{~nm}$ detection. 
From the photolysis studies, it is apparent that the formation of DEACM alcohol 4 can also be monitored. However, thio-DEACM 7 was not visible in the HPLC-UV analyses. We speculated that this is due to its low solubility in water. Therefore, aliquots from uncaging experiments were extracted with DCM (in which thio-DEACM alcohol 7 is soluble) after irradiation and analyzed by HPLC-UV. Significant amounts of thio-DEACM 7 were identified (Supplementary Figure S18).

\section{Discussion}

Thio-DEACM is a recent addition to the family of coumarin-derived photocages. It has previously been used to cage carboxylic acids [30-32] and carbamates as amine precursors [23,33]. However, it had not been introduced to phosphates. Here, we disclosed a synthetic procedure based on P-amidite chemistry to introduce the thio-DEACM photocage to adenosine oligophosphates (ADP, ATP, AP 4 ). This procedure will also be useful to introduce the thio-DEACM photocage to any phosphoric anhydride based on the modularity of the approach. We obtained small quantities of the DEACM-caged nucleotides as well, which could be readily separated by chromatography. This enabled us to directly compare the performance of the widely used DEACM photocage with thio-DEACM. We found that thio-DEACM has several advantages in uncaging experiments compared to DEACM. First, there is a significant red-shift in the UV-Vis absorption of about $90 \mathrm{~nm}$, also predicted by our DFT calculations. Moreover, we find a largely reduced fluorescence quantum yield of thio-DEACM, which translated into a largely increased photouncaging efficiency. Importantly, thio-DEACM can be removed with commercial LEDs both at 490 and $400 \mathrm{~nm}$, while DEACM is only cleaved at $400 \mathrm{~nm}$. Even at this wavelength, the thio-DEACM photocage is cleaved much more rapidly than its oxygen-containing sibling. We demonstrated the utility of this behavior by selective uncaging of nucleotides mixtures. We were able to conduct the uncaging experiments at relatively high concentrations (up to $1 \mathrm{mM}$ ) without significant interference of cleavage products. Thio-DEACM is therefore an attractive choice for caging of phosphates when compared to other caging groups regularly used in combination with phosphates $[9,44]$.

\section{Conclusions}

The quest for the development of red-shifted photocages is important to provide better compatibility with biological systems [45-48] both in terms of toxicity and penetration depth of the incident light in tissues. The possibility of low-intensity light irradiation, combined with visible light activation and rapid uncaging render thio-DEACM an alternative to the widely used DEACM, when caging of phosphates is required. By substitution of a single atom from $\mathrm{O}$ to $\mathrm{S}$, the coumarin scaffold is largely improved with regards to photocleavage properties, while retaining its stability in aqueous solution when attached to phosphoric anhydrides. Moreover, thio-DEACM shows no toxicity in live animals after photocleavage as already demonstrated in previous studies [23]. The chemistry that has been introduced here is highly modular and will help to install thio-DEACM on a range of phosphate-containing signaling molecules that can then be studied in live cell experiments.

Supplementary Materials: The following are available online. Figure S1. UV-absorbance and fluorescence spectra of DEACM-caged ADP in $\mathrm{H}_{2} \mathrm{O}$. Figure S2. UV-absorbance and fluorescence spectra of thio-DEACM-caged ADP in $\mathrm{H}_{2} \mathrm{O}$. Figure S3. UV-absorbance and fluorescence spectra of DEACM in methanol. Figure S4. UV-absorbance and fluorescence spectra of thio-DEACM in methanol. Figure S5. UV-absorbance and fluorescence spectra of DEACM-caged $\mathrm{AP}_{4}$ in $\mathrm{H}_{2} \mathrm{O}$. Figure S6. UV-absorbance and fluorescence spectra of thio-DEACM-caged $\mathrm{AP}_{4}$ in $\mathrm{H}_{2} \mathrm{O}$. Figure S7. Fluorescence quantum yield of photocages and photocaged compounds. Figure S8. Plotted spectra. Figure S9. Stability of thio-DEACM-caged nucleotides in the dark within $24 \mathrm{~h}$. Figure S10. LED irradiation for uncaging experiments, $400 \mathrm{~nm}$ and $490 \mathrm{~nm}$. Figure S11. Uncaging process of thio-DEACM-caged ATP monitored by ${ }^{31} \mathrm{P}$ proton coupled NMR. Figure S12. CE-MS identification of uncaged ATP. Figure S13. HPLC analysis of the uncaging of thio-DEACM-caged ATP at $490 \mathrm{~nm}$. Figure S14. HPLC analysis of the uncaging of thio-DEACM-caged ADP at $490 \mathrm{~nm}$. Figure S15. HPLC analysis of the uncaging of thio-DEACM-caged $\mathrm{AP}_{4}$ at $490 \mathrm{~nm}$. Figure S16. DEACM-caged nucleotides photolysis at $490 \mathrm{~nm}$ within $4 \mathrm{~min}$. Figure S17. HPLC analysis of the uncaging of DEACM- and thio-DEACM-caged ATP at 400nm. Figure S18. HPLC analysis of uncaged product (thio-DEACM). Table S1. Calculated Cartesian Coordinates for Coumarin_O on the B3LYP/def2-TZVPP level of theory. Table S2. Calculated Cartesian Coordinates for Coumarin_S on the B3LYP/def2-TZVPP level of theory. Table S3. Calculated Cartesian Coordinates for Coumarin_Se on the B3LYP/def2-TZVPP level of theory. 
Table S4. Data extracted from HPLC analysis of the uncaging of thio-DEACM-caged ATP at $490 \mathrm{~nm}$. Table S5. Data extracted from HPLC analysis of the uncaging of thio-DEACM-caged ADP at $490 \mathrm{~nm}$. Table S6. Data extracted from HPLC analysis of the uncaging of thio-DEACM-caged $\mathrm{AP}_{4}$ at $490 \mathrm{~nm}$. Table S7. Data extracted from HPLC analysis of the uncaging of DEACM- and thio-DEACM-caged ATP at 400nm. Scheme S1. Synthesis of DEACM.

Author Contributions: Conceptualization, supervision, methodology, writing-review and editing, project administration, funding acquisition H.J.J.; validation, formal analysis, investigation, data curation, writing-original draft preparation, J.M.; methodology, formal analysis, data curation, investigation, writing—review and editing, A.R.; software, supervision, writing-original draft preparation, writing-review and editing, D.W.; formal analysis, resources, writing-review and editing, T.D., D.Q., M.H., T.H., C.P., D.B.; methodology, software, validation, investigation, supervision, writing-review and editing, S.R.; writing-review and editing, B.E. All authors have read and agreed to the published version of the manuscript.

Funding: The research was funded by China Scholarship Council. Funded by the Deutsche Forschungsgemeinschaft (DFG, German Research Foundation) under Germany's Excellence Strategy-EXC-2193/ 1-390951807. Generous support by the German Research foundation (ES 361/4-1, INST 40/467-1 FUGG) and the state of Baden-Württemberg through bwHPC is gratefully acknowledged. The article processing charge was funded by the Baden-Württemberg Ministry of Science, Research and Art and the University of Freiburg in the funding programme Open Access Publishing.

Acknowledgments: We would like to thank Paraskevi Fouka, Sandra Moser for proof reading the manuscript, Jay S. Siegel and Richard Krieger for helpful discussions.

Conflicts of Interest: The authors declare no conflict of interest. The funders had no role in the design of the study; in the collection, analyses, or interpretation of data; in the writing of the manuscript, or in the decision to publish the results.

\section{References}

1. Mayer, G.; Heckel, A. Biologically active molecules with a "light switch". Angew. Chem. Int. Ed. 2006, 45, 4900-4921. [CrossRef]

2. Kaplan, J.H.; Forbush, B.; Hoffman, J.F. Rapid Photolytic Release of Adenosine 5'-Triphosphate from a Protected Analogue: Utilization by the Na:K Pump of Human Red Blood Cell Ghosts. Biochemistry 1978, 17, 1929-1935. [CrossRef] [PubMed]

3. Hansen, M.J.; Velema, W.A.; Lerch, M.M.; Szymanski, W.; Feringa, B.L. Wavelength-selective cleavage of photoprotecting groups: Strategies and applications in dynamic systems. Chem. Soc. Rev. 2015, 44, 3358-3377. [CrossRef] [PubMed]

4. Olson, J.P.; Banghart, M.R.; Sabatini, B.L.; Ellis-Davies, G.C.R. Spectral evolution of a photochemical protecting group for orthogonal two-color uncaging with visible light. J. Am. Chem. Soc. 2013, 135, 15948-15954. [CrossRef]

5. Bojtár, M.; Kormos, A.; Kis-Petik, K.; Kellermayer, M.; Kele, P. Green-Light Activatable, Water-Soluble Red-Shifted Coumarin Photocages. Org. Lett. 2019, 21, 9410-9414. [CrossRef] [PubMed]

6. Schade, B.; Hagen, V.; Schmidt, R.; Herbrich, R.; Krause, E.; Eckardt, T.; Bendig, J. Deactivation behavior and excited-state properties of (coumarin-4-yl)methyl derivatives. 1. Photocleavage of (7-methoxycoumarin4-yl)methyl- caged acids with fluorescence enhancement. J. Org. Chem. 1999, 64, 9109-9117. [CrossRef]

7. Schmidt, R.; Geissler, D.; Hagen, V.; Bendig, J. Kinetics study of the photocleavage of (coumarin-4-yl)methyl esters. J. Phys. Chem. A 2005, 109, 5000-5004. [CrossRef]

8. Schmidt, R.; Geissler, D.; Hagen, V.; Bendig, J. Mechanism of photocleavage of (coumarin-4-yl)methyl esters. J. Phys. Chem. A 2007, 111, 5768-5774. [CrossRef]

9. Pavlovic, I.; Thakor, D.T.; Vargas, J.R.; McKinlay, C.J.; Hauke, S.; Anstaett, P.; Camunã, R.C.; Bigler, L.; Gasser, G.; Schultz, C.; et al. Cellular delivery and photochemical release of a caged inositol-pyrophosphate induces PH-domain translocation in cellulo. Nat. Commun. 2016, 7, 1-8. [CrossRef]

10. Nadler, A.; Yushchenko, D.A.; Müller, R.; Stein, F.; Feng, S.; Mulle, C.; Carta, M.; Schultz, C. Exclusive photorelease of signalling lipids at the plasma membrane. Nat. Commun. 2015, 6, 1-10. [CrossRef]

11. Wagner, N.; Stephan, M.; Höglinger, D.; Nadler, A. A Click Cage: Organelle-Specific Uncaging of Lipid Messengers. Angew. Chem. Int. Ed. 2018, 57, 13339-13343. [CrossRef] [PubMed]

12. Kantevari, S.; Matsuzaki, M.; Kanemoto, Y.; Kasai, H.; Ellis-Davies, G.C.R. Two-color, two-photon uncaging of glutamate and GABA. Nat. Methods 2010, 7, 123-125. [CrossRef] [PubMed]

13. Amatrudo, J.M.; Olson, J.P.; Lur, G.; Chiu, C.Q.; Higley, M.J.; Ellis-Davies, G.C.R. Wavelength-selective oneand two-photon uncaging of Gaba. ACS Chem. Neurosci. 2014, 5, 64-70. [CrossRef] [PubMed] 
14. Olson, J.P.; Kwon, H.B.; Takasaki, K.T.; Chiu, C.Q.; Higley, M.J.; Sabatini, B.L.; Ellis-Davies, G.C.R. Optically selective two-photon uncaging of glutamate at $900 \mathrm{~nm}$. J. Am. Chem. Soc. 2013, 135, 5954-5957. [CrossRef] [PubMed]

15. Richers, M.T.; Amatrudo, J.M.; Olson, J.P.; Ellis-Davies, G.C.R. Cloaked Caged Compounds: Chemical Probes for Two-Photon Optoneurobiology. Angew. Chem. Int. Ed. 2017, 56, 193-197. [CrossRef]

16. Rovira, A.; Gandioso, A.; Goñalons, M.; Galindo, A.; Massaguer, A.; Bosch, M.; Marchán, V. Solid-Phase Approaches for Labeling Targeting Peptides with Far-Red Emitting Coumarin Fluorophores. J. Org. Chem. 2019, 84, 1808-1817. [CrossRef] [PubMed]

17. Novohradsky, V.; Rovira, A.; Hally, C.; Galindo, A.; Vigueras, G.; Gandioso, A.; Svitelova, M.; Bresolí-Obach, R.; Kostrhunova, H.; Markova, L.; et al. Towards Novel Photodynamic Anticancer Agents Generating Superoxide Anion Radicals: A Cyclometalated IrIII Complex Conjugated to a Far-Red Emitting Coumarin. Angew. Chem. Int. Ed. 2019, 58, 6311-6315. [CrossRef] [PubMed]

18. Gandioso, A.; Palau, M.; Nin-Hill, A.; Melnyk, I.; Rovira, C.; Nonell, S.; Velasco, D.; García-Amorós, J.; Marchán, V. Sequential Uncaging with Green Light can be Achieved by Fine-Tuning the Structure of a Dicyanocoumarin Chromophore. ChemistryOpen 2017, 6, 375-384. [CrossRef]

19. Gandioso, A.; Contreras, S.; Melnyk, I.; Oliva, J.; Nonell, S.; Velasco, D.; García-Amorós, J.; Marchán, V. Development of Green/Red-Absorbing Chromophores Based on a Coumarin Scaffold That Are Useful as Caging Groups. J. Org. Chem. 2017, 82, 5398-5408. [CrossRef]

20. Gandioso, A.; Bresolí-Obach, R.; Nin-Hill, A.; Bosch, M.; Palau, M.; Galindo, A.; Contreras, S.; Rovira, A.; Rovira, C.; Nonell, S.; et al. Redesigning the Coumarin Scaffold into Small Bright Fluorophores with Far-Red to Near-Infrared Emission and Large Stokes Shifts Useful for Cell Imaging. J. Org. Chem. 2018, 83, 1185-1195. [CrossRef]

21. Gandioso, A.; Palau, M.; Bresolí-Obach, R.; Galindo, A.; Rovira, A.; Bosch, M.; Nonell, S.; Marchán, V. High Photostability in Nonconventional Coumarins with Far-Red/NIR Emission through Azetidinyl Substitution. J. Org. Chem. 2018, 83, 11519-11531. [CrossRef] [PubMed]

22. Bassolino, G.; Nançoz, C.; Thiel, Z.; Bois, E.; Vauthey, E.; Rivera-Fuentes, P. Photolabile coumarins with improved efficiency through azetidinyl substitution. Chem. Sci. 2018, 9, 387-391. [CrossRef] [PubMed]

23. Fournier, L.; Gauron, C.; Xu, L.; Aujard, I.; Le Saux, T.; Gagey-Eilstein, N.; Maurin, S.; Dubruille, S.; Baudin, J.B.; Bensimon, D.; et al. A blue-absorbing photolabile protecting group for in vivo chromatically orthogonal photoactivation. ACS Chem. Biol. 2013, 8, 1528-1536. [CrossRef] [PubMed]

24. Guan, Z.; Inscho, E.W. Role of adenosine $5^{\prime}$-triphosphate in regulating renal microvascular function and in hypertension. Hypertension 2011, 58, 333-340. [CrossRef] [PubMed]

25. Thirlwell, H.; Corrie, J.E.; Reid, G.P.; Trentham, D.R.; Ferenczi, M.A. Kinetics of relaxation from rigor of permeabilized fast-twitch skeletal fibers from the rabbit using a novel caged ATP and apyrase. Biophys. J. 1994, 67, 2436-2447. [CrossRef]

26. Sokolov, V.S.; Apell, H.J.; Corrie, J.E.T.; Trentham, D.R. Fast transient currents in Na,K-ATPase induced by ATP concentration jumps from the P3-[1-(3',5'-dimethoxyphenyl)-2-phenyl-2-oxo]ethyl ester of ATP. Biophys. J. 1998, 74, 2285-2298. [CrossRef]

27. Park, C.H.; Givens, R.S. New photoactivated protecting groups. 6. p-Hydroxyphenacyl: A phototrigger for chemical and biochemical probes. J. Am. Chem. Soc. 1997, 119, 2453-2463. [CrossRef]

28. Geißler, D.; Kresse, W.; Wiesner, B.; Bendig, J.; Kettenmann, H.; Hagen, V. DMACM-caged adenosine nucleotides: Ultrafast phototriggers for ATP, ADP, and AMP activated by long-wavelength irradiation. ChemBioChem 2003, 4, 162-170. [CrossRef]

29. Pinheiro, A.; Baptistap, P.; Lima, J.C. Light activation of transcription: Photocaging of nucleotides for control over RNA polymerization. Nucleic Acids Res. 2008, 36. [CrossRef]

30. Fonseca, A.S.C.; Soares, A.M.S.; Gonçalves, M.S.T.; Costa, S.P.G. Thionated coumarins and quinolones in the light triggered release of a model amino acid: Synthesis and photolysis studies. Tetrahedron 2012, 68, 7892-7900. [CrossRef]

31. Fournier, L.; Aujard, I.; Le Saux, T.; Maurin, S.; Beaupierre, S.; Baudin, J.B.; Jullien, L. Coumarinylmethyl caging groups with redshifted absorption. Chem. A Eur. J. 2013, 19, 17494-17507. [CrossRef] [PubMed]

32. Chen, Z.; Sun, W.; Butt, H.J.; Wu, S. Upconverting-nanoparticle-assisted photochemistry induced by low-intensity near-infrared light: How low can we go? Chem. A Eur. J. 2015, 21, 9165-9170. [CrossRef] [PubMed] 
33. Manna, D.; Maji, B.; Gangopadhyay, S.A.; Cox, K.J.; Zhou, Q.; Law, B.K.; Mazitschek, R.; Choudhary, A. A Singular System with Precise Dosing and Spatiotemporal Control of CRISPR-Cas9. Angew. Chem. Int. Ed. 2019, 58, 6285-6289. [CrossRef] [PubMed]

34. Weinrich, T.; Gränz, M.; Grünewald, C.; Prisner, T.F.; Göbel, M.W. Synthesis of a Cytidine Phosphoramidite with Protected Nitroxide Spin Label for EPR Experiments with RNA. Eur. J. Org. Chem. 2017, 2017, 491-496. [CrossRef]

35. Caruthers, M.H. Chemical Synthesis of DNA and DNA Analogues. Acc. Chem. Res. 1991, 24, $278-284$. [CrossRef]

36. Hofer, A.; Cremosnik, G.S.; Müller, A.C.; Giambruno, R.; Trefzer, C.; Superti-Furga, G.; Bennett, K.L.; Jessen, H.J. A Modular Synthesis of Modified Phosphoanhydrides. Chem. A Eur. J. 2015, 21, 10116-10122. [CrossRef]

37. Singh, J.; Ripp, A.; Haas, T.M.; Qiu, D.; Keller, M.; Wender, P.A.; Siegel, J.S.; Baldridge, K.K.; Jessen, H.J. Synthesis of Modified Nucleoside Oligophosphates Simplified: Fast, Pure, and Protecting Group Free. J. Am. Chem. Soc. 2019, 141, 15013-15017. [CrossRef]

38. Haas, T.M.; Ebensperger, P.; Eisenbeis, V.B.; Nopper, C.; Dürr, T.; Jork, N.; Steck, N.; Jessen-Trefzer, C.; Jessen, H.J. Magic spot nucleotides: Tunable target-specific chemoenzymatic synthesis. Chem. Commun. 2019, 55, 5339-5342. [CrossRef]

39. Lee, D.R.; Lee, K.H.; Shao, W.; Kim, C.L.; Kim, J.; Lee, J.Y. Heavy Atom Effect of Selenium for Metal-Free Phosphorescent Light-Emitting Diodes. Chem. Mater. 2020, 32, 2583-2592. [CrossRef]

40. Becke, A.D. Density-functional thermochemistry. III. The role of exact exchange. J. Chem. Phys. 1993, 98, 5648-5652. [CrossRef]

41. Stephens, P.J.; Devlin, F.J.; Chabalowski, C.F.; Frisch, M.J. Ab Initio Calculation of Vibrational Absorption and Circular Dichroism Spectra Using Density Functional Force Fields. J. Phys. Chem. 1994, 98, 11623-11627. [CrossRef]

42. Weigend, F. Accurate Coulomb-fitting basis sets for H to Rn. Phys. Chem. Chem. Phys. 2006, 8, 1057-1065. [CrossRef] [PubMed]

43. Weigend, F.; Ahlrichs, R. Balanced basis sets of split valence, triple zeta valence and quadruple zeta valence quality for H to Rn: Design and assessment of accuracy. Phys. Chem. Chem. Phys. 2005, 7, 3297-3305. [CrossRef] [PubMed]

44. Mordhorst, S.; Singh, J.; Mohr, M.K.F.; Hinkelmann, R.; Keppler, M.; Jessen, H.J.; Andexer, J.N. Several Polyphosphate Kinase 2 Enzymes Catalyse the Production of Adenosine 5'-Polyphosphates. ChemBioChem 2019, 20, 1019-1022. [CrossRef] [PubMed]

45. Bittner, T.; Wittwer, C.; Hauke, S.; Wohlwend, D.; Mundinger, S.; Dutta, A.K.; Bezold, D.; Dürr, T.; Friedrich, T.; Schultz, C.; et al. Photolysis of Caged Inositol Pyrophosphate InsP8Directly Modulates Intracellular Ca2+Oscillations and Controls C2AB Domain Localization. J. Am. Chem. Soc. 2020, 142, 10606-10611. [CrossRef]

46. Peterson, J.A.; Wijesooriya, C.; Gehrmann, E.J.; Mahoney, K.M.; Goswami, P.P.; Albright, T.R.; Syed, A.; Dutton, A.S.; Smith, E.A.; Winter, A.H. Family of BODIPY Photocages Cleaved by Single Photons of Visible/Near-Infrared Light. J. Am. Chem. Soc. 2018, 140, 7343-7346. [CrossRef]

47. Kand, D.; Liu, P.; Navarro, M.X.; Fischer, L.J.; Rousso-Noori, L.; Friedmann-Morvinski, D.; Winter, A.H.; Miller, E.W.; Weinstain, R. Water-Soluble BODIPY Photocages with Tunable Cellular Localization. J. Am. Chem. Soc. 2020, 142, 4970-4974. [CrossRef]

48. Shrestha, P.; Dissanayake, K.C.; Gehrmann, E.J.; Wijesooriya, C.S.; Mukhopadhyay, A.; Smith, E.A.; Winter, A.H. Efficient Far-Red/Near-IR Absorbing BODIPY Photocages by Blocking Unproductive Conical Intersections. J. Am. Chem. Soc. 2020, 142, 15505-15512. [CrossRef]

Publisher's Note: MDPI stays neutral with regard to jurisdictional claims in published maps and institutional affiliations. 